\title{
La 6 a Olimpíada Internacional de Ciencias de la Tierra en Argentina: crónica de una experiencia compleja
}

\author{
José Sellés-Martínez \\ Dpto. de Ciencias Geológicas y CEFIEC, \\ Facultad de Ciencias Exactas y Naturales, \\ Universidad de Buenos Aires, Argentina. \\ pepe@gl.fcen.uba.ar
}

\begin{abstract}
The International Earth Sciences Olympiad (IESO) is a young event with just a few years of tradition. However, they are growing constantly after the initial impulse given by the countries of south-eastern Asia. The $6^{\text {th }}$ edition of the event, initially planned to take place in Japan in 2012, was moved urgently after the earthquake and tsunami that hit the country in 2011. Although Argentina has no Earth Sciences as a secondary school curricular subject, the fact that the country was present since the beginning in the institutions that organized the event (IGEO and IESO), made it possible to take the decision to move the event to Argentina. The organization of the Olympiad became complex due to administrative and political factors affecting the country and the hosting Province of Buenos Aires (where it took place); nevertheless, the results were very highly satisfactory thanks to the enthusiasm and spirit of cooperation of the 18 participating teams.Citation: Seles-Martínez J. 2014. Consequências das Olimpíadas Internacionais de Geociências para o ensino argentino.Terræ Didatica, 10(3):260-273. http://www.ige.unicamp.br/terraedidatica/.
\end{abstract}

KEYWORDS: International Olympiads; Geology; Earth Sciences; Science Education.

RESUMEN Las Olimpíadas Internacionales de Ciencias de la Tierra (IESO) cuentan con muy pocos años de tradición, sin embargo, su desarrollo, impulsado fuertemente por los países del sudeste asiático, se encuentra en franca expansión. La $6^{a}$ edición del evento, que debía realizarse en el año 2012 en Japón, debió trasladarse, debido a las consecuencias del sismo y tsunami del 2011 a la Argentina. Si bien este país no cuenta con tradición en la enseñanza de las Ciencias de la Tierra a nivel secundario, su participación activa en las instituciones madres de la Olimpíada, la IGEO y la IESO, posibilitó esta circunstancia. La organización de la Olimpíada estuvo signada por circunstancias administrativas y económicas complejas a nivel de los gobiernos del país y de la Provincia de Buenos Aires, donde finalmente se realizó. A pesar de ello, y gracias a la colaboración y el entusiasmo de las 18 delegaciones participantes, el evento resultó altamente satisfactorio.

PALAVRAS-CHAVES: Olimpiadas internacionales; Geología; Ciencias de la Tierra; Enseñanza de las Ciencias. 


\section{Introducción}

las Olimpíadas Internacionales de Matemáticas, decanas de este tipo de actividades, cuentan ya con más de medio siglo de historia. A lo largo de los años, otras disciplinas fueron incorporándose a la lista de competencias internacionales tanto en los niveles de estudios secundarios como, en algunos casos (Olimpíadas de Computación) a nivel universitario. La enseñanza de las Ciencias de la Tierra, si bien menos intensiva con respecto a otras disciplinas, se encuentra también presente en los curricula de numerosos países y esto llevó a los docentes y gestores de políticas educativas de algunos de ellos a plantearse por qué no podían organizarse olimpíadas de Ciencias de la Tierra, tanto a nivel nacional como también un evento de alcance internacional. La justificación más evidente es que los estudiantes, y la sociedad en general, deben conocer las Ciencias de la Tierra no sólo por su interés académico, sino porque su vida cotidiana está estrechamente ligada a ellas y lo estará cada vez más en el futuro, y el desarrollo de competencias a nivel nacional e internacionales visto como un incentivo al aprendizaje de la disciplina y contribuye a desarrollar nuevas habilidades y, sobre todo, nuevas actitudes en temas tan críticos como el cuidado del medio ambiente, el uso de los recursos no renovables, etc. Por otra parte, es también importante que, en una sociedad cada vez más internacionalizada, los jóvenes estudiantes conozcan, comprendan y compartan las diferentes identidades culturales que los participantes provenientes de otros países representan. La Olimpíada es un ámbito excepcional que proporciona ocasión de conocimiento mutuo e inicio de relaciones de amistad entre jóvenes de todo el mundo. Estos breves conceptos condujeron a la creación de este evento, la Olimpíada Internacional de Ciencias de la Tierra, IESO por sus siglas en inglés, cuya historia, características generales y realización en la Argentina en 2012 se describen en esta contribución. Se ha hecho énfasis en los detalles de organización en la consideración de que el informe aquí consignado pueda ser de referencia y utilidad a aquéllos países que nunca hayan participado del evento y deseen conocer sus características, de modo de contar con la información necesaria para encarar su participación como país y como delegación y la preparación de sus estudiantes en las disciplinas integradas bajo la denominación "ciencias de la Tierra".

\section{Breve historia de las IESO}

En el año 2003 tuvo lugar en la ciudad de Calgary (Canadá) la 4a Conferencia sobre Educación en Geociencias (GeoSciEd), organizada por la Internacional Geoscience Education Organization (IGEO) Allí, en el marco de una reunión de los representantes de todos los países miembros, el representante de Corea propone la realización de un futuro encuentro para estudiar las posibilidades de organizar una Olimpíada Internacional de Ciencias de la Tierra. Algunos representantes se muestran favorables a la realización de una Olimpíada Internacional, mientras que otros se muestran escépticos o incluso se oponen a ese tipo de eventos por considerar que promueven exclusivamente la competencia sin ningún tipo de beneficio real educativo para los estudiantes.

Finalmente los países que muestran interés por la idea son invitados a reunirse en Seúl (Corea) en el mes de Noviembre del año 2004 y allí se sientan las bases para la organización de las Olimpíadas Internacionales de Ciencias de la Tierra, sus características y los contenidos disciplinares a ser considerados. Las presentaciones realizadas en esa reunión han sido publicadas (Kim y Kwak, 2004) y se remite al lector interesado tanto en las características de la enseñanza de las Ciencias de la Tierra en los países participantes, como en los detalles de las propuestas de contenidos y organización de las futuras IESO a esa publicación para mayores detalles. El país anfitrión, quizás el más entusiasta en que el evento internacional tenga lugar, se ofrece como organizador de la primera IESO, proponiendo la misma para el año 2007. En esa primera Olimpíada participan 8 países, número que va incrementándose a medida que se realizan nuevas Olimpíadas en los años subsiguientes, si bien con algunos altibajos. La segunda IESO se realiza en 2008 en Filipinas, con la participación de 7 países. Taiwán, en 2009 convoca ya a 17 países, un número similar, 18, concurre a la IESO de 2010 en Indonesia y en el 2011 el número de participantes en la IESO que tiene lugar en Italia llega a 33. Finalmente, en 2012 la 6 a IESO se realiza en Argentina, participando del evento 19 países. Lamentablemente, por diferentes razones, Bangladesh, Bolivia, Colombia, Chile y Nigeria, que estaban muy interesados en participar en Argentina no pudieron concurrir. Es importante señalar que por razones que se expondrán al desarrollar con mayor detalle la $6^{a}$ Olimpíada, y a diferencia de los eventos anteriores, el país anfitrión 
no cubrió gastos de ninguna delegación extranjera, lo que explica en gran parte la caída en el número de participantes con respecto al evento previo. En la Tabla 1 se reseñan los países participantes en cada una de las ediciones.

\section{La organización de cada IESO}

Las IESOs son una de las actividades principales, si no la principal, de la IGEO. En ese contexto, esta institución es responsable de la constitución y funcionamiento del Comité Internacional para las Olimpíadas (International IESO Advisory Committee) del que participan la Presidencia de la IGEO y miembros designados por ella y el Comité Coordinador Internacional (International Coordinating Committee) constituido por la presidencia de la IGEO y los organizadores de eventos IESO recientes y próximos. El trabajo de organización, difusión, etc. recae totalmente en el Comité Organizador Local propio de cada país, el que es elegido en absoluta libertad por el representante del mismo en la IESO y es apoyado por el Comité Coordinador.

Los países que desean participar deben registrarse e informarse de las normas que regulan las IESO, sobre todo aquéllas referentes a las edades máximas y condiciones que deben cumplir los alumnos participantes. Para ello se dispone de amplia información en la página web de cada evento y, en una página propia de las IESO (http://www. ieso-info.org), en la que se acumula la información histórica.

Cada país puede participar enviando:

1. Un equipo completo, constituido por 4 estudiantes y 2 mentores

2. Un equipo reducido, con menos de 4 estudiantes y sólo 1 mentor

3. Observadores en el número que deseen

4. Acompañantes e invitados (estudiantes o adultos) en el número que deseen

Con respecto al presupuesto de la IESO cabe señalar como características fundamentales que el país organizador cubre los gastos de alojamiento, alimentación y desplazamientos locales y que debe hacerse cargo de conseguir esos fondos por sí mismo, ya que la IGEO no solventa gastos de organización. Cada país participante debe hacerse cargo, por su parte, del costo de los pasajes aéreos y de los gastos de inscripción. En este último rubro es muy importante tener en cuenta que cada equipo representante abona el costo de la inscripción como país participante, pero cada individuo adicional (observadores, acompañantes e invitados) debe abonar una inscripción cuyo costo es significativo y que, generalmente no es menor al costo real que genera en alojamiento, alimentación y traslado. La participación de observadores es sin embargo deseada e importante, ya que los mismos colaboran activamente en muchas de las tareas durante el desarrollo del evento. Con respecto a los estudiantes que participan como invitados, si bien los mismos realizan las pruebas tal como los miembros del equipo "oficial", sus resultados no son tenidos en cuenta en el momento de decidirse los premios.

\section{El estandarte olímpico}

Con motivo de la realización de la $1^{\mathrm{a}}$ Olimpíada, las autoridades del Comité Organizador Local, confeccionaron un estandarte con el logo de ese evento. Este estandarte es transferido al final de cada acto de premiación al representante del país que hospedará el evento al año siguiente, en un lejano recuerdo del viaje de la antorcha en el caso de las olimpíadas deportivas. En la Figura 1 puede verse el momento en que el representante de Argentina recibe el estandarte de manos del organizador del evento en Italia, Dr. Roberto Greco y del Dr. Moon Young Song, en ese momento

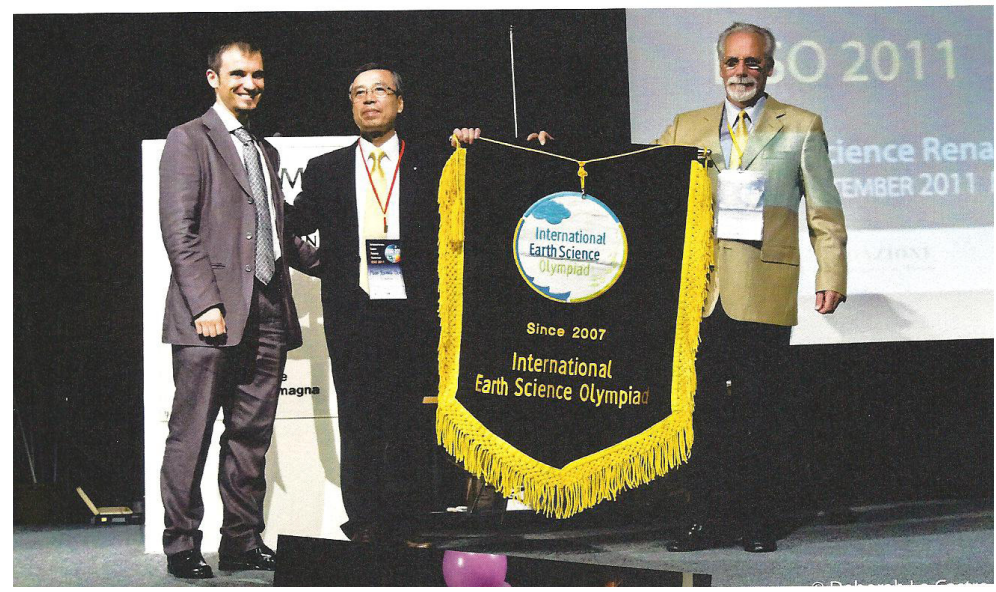

Figura 1. Transferencia del estandarte de las Olimpíadas de Italia a Argentina 
Tabla 1. Países participantes en cada una de las IESO

\begin{tabular}{|c|c|c|c|c|c|c|}
\hline $\begin{array}{c}\text { Año } \\
\text { País organizador }\end{array}$ & $\begin{array}{l}2007 \\
\text { Corea }\end{array}$ & $\begin{array}{c}2008 \\
\text { Filipinas }\end{array}$ & $\begin{array}{c}2009 \\
\text { Taiwán }\end{array}$ & $\begin{array}{c}2010 \\
\text { Indonesia }\end{array}$ & $\begin{array}{c}2011 \\
\text { Italia }\end{array}$ & $\begin{array}{c}2012 \\
\text { Argentina }\end{array}$ \\
\hline Participantes & & & & & & \\
\hline Argentina & & & $\mathrm{ob}$ & & $\mathrm{ob}$ & Org. \\
\hline Alemania & & & & & & $\mathrm{x}$ \\
\hline Australia & & & & & $\mathrm{x}$ & $\mathrm{x}$ \\
\hline Austria & & & & & $\mathrm{x}$ & $\mathrm{x}$ \\
\hline Belarus & & & & & $\mathrm{x}$ & $\mathrm{x}$ \\
\hline Brasil & & & & & & $\mathrm{x}$ \\
\hline Camboya & & & $\mathrm{x}$ & $\mathrm{x}$ & $\mathrm{x}$ & \\
\hline Corea & $\mathrm{x}$ & $\mathrm{x}$ & $\mathrm{x}$ & $\mathrm{x}$ & $\mathrm{x}$ & $\mathrm{x}$ \\
\hline Chipre & & & & & $\mathrm{ob}$ & \\
\hline España & & & & & $\mathrm{x}$ & $\mathrm{x}$ \\
\hline Estados Unidos & $\mathrm{x}$ & $\mathrm{x}$ & $\mathrm{x}$ & $\mathrm{x}$ & $\mathrm{x}$ & $\mathrm{x}$ \\
\hline Etiopía & & & & $\mathrm{ob}$ & & \\
\hline Francia & & & $\mathrm{ob}$ & $\mathrm{ob}$ & $\mathrm{x}$ & $\mathrm{x}$ \\
\hline Filipinas & $\mathrm{x}$ & $\mathrm{x}$ & $\mathrm{x}$ & $\mathrm{x}$ & $\mathrm{x}$ & \\
\hline Honduras & & & & & $\mathrm{x}$ & \\
\hline India & $\mathrm{x}$ & & $\mathrm{x}$ & $\mathrm{x}$ & $\mathrm{x}$ & $\mathrm{x}$ \\
\hline Indonesia & $\mathrm{x}$ & $\mathrm{ob}$ & $\mathrm{x}$ & $\mathrm{x}$ & $\mathrm{x}$ & $\mathrm{x}$ \\
\hline Israel & & & & & $\mathrm{x}$ & \\
\hline Italia & & & $\mathrm{x}$ & $\mathrm{x}$ & $\mathrm{x}$ & $\mathrm{x}$ \\
\hline Japón & $\mathrm{ob}$ & $\mathrm{x}$ & $\mathrm{x}$ & $\mathrm{x}$ & $\mathrm{x}$ & $\mathrm{x}$ \\
\hline Kirgistán & & & & & $\mathrm{x}$ & \\
\hline Kuwait & & & & $\mathrm{x}$ & $\mathrm{x}$ & $\mathrm{x}$ \\
\hline Malasia & & & & & $\mathrm{ob}$ & \\
\hline Malawi & & & & & $\mathrm{x}$ & \\
\hline Maldivas & & & & $\mathrm{x}$ & & \\
\hline Malta & & & & & $\mathrm{ob}$ & \\
\hline Mongolia & $\mathrm{x}$ & & & & & \\
\hline Nepal & & & $\mathrm{x}$ & $\mathrm{x}$ & $\mathrm{x}$ & \\
\hline Noruega & & & & & $\mathrm{x}$ & \\
\hline Pakistán & & & & & $\mathrm{ob}$ & \\
\hline Reino Unido & & & $\mathrm{x}$ & & & \\
\hline Rumania & & & & $\mathrm{x}$ & $\mathrm{x}$ & $\mathrm{x}$ \\
\hline Rusia & & & & $\mathrm{x}$ & $\mathrm{x}$ & $\mathrm{x}$ \\
\hline Singapur & & $\mathrm{x}$ & $\mathrm{x}$ & & $\mathrm{x}$ & \\
\hline Sri Lanka & & & $\mathrm{x}$ & $\mathrm{x}$ & $\mathrm{x}$ & \\
\hline Sudáfrica & & & & & $\mathrm{ob}$ & \\
\hline Tailandia & & & $\mathrm{x}$ & $\mathrm{x}$ & $\mathrm{x}$ & $\mathrm{x}$ \\
\hline Taiwán & $\mathrm{x}$ & $\mathrm{x}$ & $\mathrm{x}$ & $\mathrm{x}$ & $\mathrm{x}$ & $\mathrm{x}$ \\
\hline Ucrania & & & $\mathrm{ob}$ & $\mathrm{x}$ & $\mathrm{x}$ & $\mathrm{x}$ \\
\hline
\end{tabular}

Ob. : observador; $\mathrm{x}$ : equipo representativo 
presidente del Comité Internacional.

\section{Los lemas de las Olimpíadas}

Dado que una de las misiones de las IESO es crear conciencia de la importancia de las Ciencias de la Tierra en la sociedad, a partir de la $2^{\mathrm{a}}$ Olimpíada se introdujo un lema o tema guía del evento, aunque es importante destacar, esto no se vio reflejado en la composición de las preguntas y ejercicios de cada Olimpíada. Los lemas adoptados fueron:

2008: "Cooptation". Enfrentar El Cambio Climático (Aclaración. Cooptation es la contracción de cooperation y competition)

2009: El Hombre Y El Medio Ambiente

2010:El Presente Es La Clave Del Futuro

2011: Renacimiento En Las Ciencias De La Tierra. Ciencia, Medio Ambiente y Arte

2012: Energía, Agua y Minerales Para El Desarrollo Sustentable

\section{Las "Ciencias de la Tierra" en el marco de las Olimpíadas Internacionales}

Una de las discusiones más relevantes que se dieron durante el proceso de organización, y que generalmente se renuevan cada año, son los temas a ser incluidos en las evaluaciones. Las razones de las discusiones son varias y van desde una filosofía general acerca de qué son las Ciencias de la Tierra a razones más prácticas respecto a si los estudiantes de cada país en particular abordan o no esos contenidos durante el proceso de su formación en la escuela secundaria. Finalmente se incluyeron como disciplinas integrantes del Syllabus a las siguientes: Geología, Ciencias de la Atmósfera, Oceanografía, Ciencias Ambientales y Astronomía, aunque el peso relativo de cada una de ellas en la composición de las pruebas es diferente y ha sido establecido por consenso en los siguientes valores: Geosfera (45\%), Atmosfera e Hidrosfera (35\%) y Astronomía $(20 \%)$. Estos porcentajes valen tanto para el examen teórico como para el práctico. El peso relativo de ambas formas de evaluación es 70\% para la prueba escrita y $30 \%$ para la práctica. Los contenidos de Ciencias Ambientales se encuentran comprendidos dentro de las preguntas de Geosfera, Atmosfera e Hidrosfera. En general se trata de que las preguntas relativas a Astronomía se circunscriban a aspectos que estén vinculados en forma directa o indirecta al planeta Tierra y no a temas espaciales sin conexión evidente con nuestro planeta ya que, por un lado carecen de interés para el enfoque de las IESO y, por otro, existen Olimpíadas específicas de Astronomía en las cuales son evaluados dichos conocimientos.

\section{Las formas de evaluar}

Una importante discusión dentro del Comité Organizador giró en torno a cómo evaluar conocimientos y habilidades (véase al respecto Kim y Kwak, 2004) y, sobre todo, un grupo de participantes estaba fuertemente convencido de que las Olimpíadas no debían servir sólo para que los estudiantes se enfrentaran y midieran conocimientos, sino también para facilitar la comunicación entre ellos y crear lazos que perduraran más allá del evento, sobre todo tomándose en consideración que las modernas tecnologías de la comunicación hacen esto fácil y accesible para la gran mayoría de los jóvenes. De tal modo, la estructura de evaluación quedó definida del siguiente modo:

1. Pruebas teóricas: Los estudiantes deben responder preguntas y resolver problemas "de escritorio" que no requieren instrumental ni observaciones prácticas.

2. Pruebas prácticas (problemas y laboratorio): Los estudiantes deben realizar descripciones, mediciones, interpretaciones y/o sacar conclusiones de sus observaciones y/o manipulaciones sobre materiales, utilizando el instrumental necesario (siempre provisto por los organizadores).

3. Es importante señalar que la preparación de las pruebas teóricas y prácticas está a cargo de una comisión ad hoc, pero que todos los países están invitados a colaborar enviando preguntas y ejercicios para ser incorporados a las pruebas. Todas las preguntas y ejercicios son analizados y discutidos (a veces muy intensamente) en una reunión del jurado internacional, ya dentro de la Olimpíada, uno o dos dìas antes de ser presentados a los participantes. Los tutores que lo desean pueden traducir las preguntas del idioma inglés al de su país y, si los alumnos responden en su lengua materna, deben traducir las respuestas para los miembros del jurado. Toda la documentación, preguntas originales, traducidas, respuestas originales y traducidas, debe ser conservada en forma escrita para su supervisión en caso de problemas o dudas.

4. El trabajo de campo en equipos internacionales. Dadas sus peculiaridades se describe en el párrafo siguiente. 


\section{El trabajo de campo en equipos internacionales}

La realización de este trabajo, llamado International Teams Field Investigation (ITFI) fue, finalmente, el modo en que logró materializarse la inclusión, en la estructura evaluativa, de una actividad que promoviera las relaciones entre los participantes. El mismo es, además y por su naturaleza, representativo de una característica intrínseca y arquetípica de las Ciencias de la Tierra: el trabajo de campo. Los organizadores deben encontrar lugares a los que llevar a los diferentes equipos y problemas que puedan resolver con la información que se les proporciona y las observaciones y datos que deben tomar in situ. Los equipos están compuestos por representantes de diferentes países y, en general, se trata que en todos ellos haya algún estudiante con dominio del inglés como lengua materna para facilitar la redacción del informe final y la preparación del Power Point, con que deben hacer públicas sus conclusiones frente a los jurados convocados al efecto, el público asistente y los miembros de los otros equipos. Si bien la organización de esta actividad no es sencilla, su impacto y eficiencia para desarrollar las comunicaciones entre los representantes de los diferentes países ha sido siempre muy alto y ha sido siempre valorada como muy positiva por los estudiantes y educadores.

Los galardones resultantes de este trabajo, luego de una serie de discusiones, no han sido finalmente validados para el otorgamiento de las medallas, pero cada miembro de los equipos que han presentado los mejores trabajos recibe un trofeo y un diploma acreditándolo.

\section{Los premios y distinciones}

La forma en que se reconocerían los méritos de los participantes fue también motivo de intercambio de opiniones y finalmente se estableció que para el otorgamiento de las medallas sólo se tendrían en cuenta los exámenes teórico y práctico y que al trabajo de campo se le entregaría un reconocimiento que no debía llevar el título de "medalla" para no crear confusiones. En el caso de la Olimpíada en Argentina se trató de un trofeo, que se describe más adelante. Todos los participantes que resultaran premiados recibirían, además de su medalla o trofeo, un diploma oficial acreditando el hecho.

El número total de medallas a otorgar es generalmente consensuado en cada IESO en función del número total de participantes, sobre un criterio general de $10 \%$ de medallas de oro, $20 \%$ de medallas de plata y $30 \%$ de medallas de bronce. Vale la pena aclarar que "oro", "plata" y "bronce" no reflejan fielmente la composición metálica de las medallas, que son realizadas generalmente en otros materiales de menor costo.

Para el Trabajo de Campo se han establecido tres premios, al "Mejor Trabajo", al "Trabajo más Creativo" y al "Equipo Mejor Integrado", esta última valoración surge de las observaciones realizadas por los jueces durante la labor de campo y en la acción de exponer las conclusiones.

\section{Documentos e información adicional acerca de las olimpíadas}

Información adicional acerca de las olimpíadas puede obtenerse en los sitios web de cada uno de los eventos realizados, en el sitio web oficial de la IESO y en el de la IGEO, cuyos URLs son los siguientes: Filipinas: http://ieso2008.org/index.php

Taiwan: http://www.ieso2009.tw/

Italia: http://www.ieso2011.unimore.it/ Argentina: http://ieso2012.gl.fcen.uba.ar/

IESO:http://www.ieso-info.org/archives/1

IGEO:http://www.geoscied.org/

\section{La olimpíada en Argentina}

\section{El porqué y el cómo}

El día 11 de Marzo del 2011, un fenómeno geológico excepcional por su magnitud y consecuencias, un sismo que alcanzó los 9 grados, afectó gravemente a gran parte de la costa oriental del Japón, provocando severísimos daños y un muy alto costo en vidas humanas. Como consecuencia de esto, los organizadores de la $6^{\mathrm{a}}$ IESO, que debía celebrarse en Japón, solicitaron al Comité Internacional ser relevados de dicha tarea y el Comité informó a sus miembros que debía encontrarse una sede alternativa a la mayor brevedad posible. Por razones de pertenencia a IGEO y a IESO, y por encontrar una solución a esta situación, se realizó un relevamiento preliminar acerca de los apoyos que podrían conseguirse en la Argentina y se propuso la misma como sede, la que fue aceptada durante la reunión plenaria de la $5^{\mathrm{a}}$ IESO en Italia, aunque ya informalmente se habían realizado algunos trámites previos. Una tarea que se inició en forma más o menos sencilla y con apoyos importantes prometidos, terminó haciéndose, sin embargo, muy compleja debido a 
los acontecimientos político-económicos que se produjeron a partir de enero del 2012. Como resultado de ello y para reducir costos, el evento debió acortarse y desplazarse al interior de la provincia de Buenos Aires, suprimiéndose las excursiones programadas y centralizándose toda la actividad en la ciudad de Olavarría, seleccionada tanto por la calidad de su entorno geológico, como por la excelente disposición de sus autoridades para colaborar en la organización del evento.

\section{Problemas vinculados a la ausencia de la disciplina en los planes de estudio de la Argentina}

La no existencia de las Ciencias de la Tierra como disciplina en los planes de estudio de las escuelas secundarias, tanto a nivel nacional como provincial, dificultó mucho la gestión de apoyo en los respectivos Ministerio y Dirección de Educación e impidió totalmente la participación de un equipo representativo de nuestro país en el evento. Cabe señalar, a modo de ejemplo, que si bien se logró que el Ministerio de Ciencias y Tecnología de la Nación apoyara la iniciativa y, con mucho esfuerzo, que el Ministerio de Educación la declarara de interés educativo, fue imposible lograr una declaratoria similar por parte de la Dirección de Educación provincial y, a pesar de haber realizado entrevistas personales con las autoridades correspondientes, nunca se obtuvo respuesta escrita a las cartas elevadas. Por el contrario, fue notable el interés y colaboración por parte de la Dirección de Turismo Provincial que asesoró respecto a las posibles sedes y facilitó el acceso a las autoridades de las ciudades de Tandil y Olavarría, seleccionadas en primera instancia como candidatas.

\section{Búsqueda de financiación}

Desde el punto de vista de las empresas e instituciones auspiciantes cabe señalar que los difíciles momentos político-económicos vividos en ese año afectaron en forma negativa la gestión de fondos. El caso más relevante lo constituye el de empresa REPSOL-YPF, que había comprometido su colaboración, nada despreciable por cierto, tanto a través de su Fundación como de su gerencia de exploración. Sin embargo, luego de la expulsión de la empresa española en el mes de enero del 2012, fue imposible conseguir colaboración de YPF. Sin embargo algunas empresas e instituciones colaboraron y fue así que el Servicio Geológico y Minero de la Argentina (SEGEMAR) se hizo cargo de la impresión de las camisetas a ser distribuidas entre los estudiantes y de algunos objetos de memorabilia que serán descriptos más adelante. La empresa Pan American Energy realizó una valiosa donación en efectivo y ARCOR colaboró con abundantes bombones y caramelos que endulzaron la semana a los participantes. Para interiorizar a las empresas sobre qué son las IESOs y porqué debían ser apoyadas económicamente, se diseñó un folleto que se imprimió a color y se remitió, junto con una carta institucional a los potenciales colaboradores, vale la pena señalar que sin mucho éxito. La gestión de los fondos del evento fue posible gracias a la colaboración institucional de la Fundación Ciencias Exactas, que puso su cuenta bancaria a disposición de los organizadores, facilitando enormemente la recepción de fondos, facturación y realización de pagos de servicios y bienes. Entre las instituciones académicas que brindaron su apoyo deben mencionarse la Sociedad Científica Argentina, la Asociación Geológica Argentina y la Asociación Argentina de Geofísicos y Geodestas. El Departamento de Ciencias Geológicas de la Facultad de Ciencias Exactas y Naturales de la Universidad de Buenos Aires (FCEyN - UBA), por su parte, facilitó todo lo posible la participación de sus miembros en la organización de diferentes aspectos del evento, incluida la asistencia de los jóvenes estudiantes que colaboraron como guías e intérpretes de los equipos visitantes.

\section{Actividades culturales asociadas}

En la planificación original se incluían numerosas actividades asociadas y preparatorias de la Olimpiada en nuestro país. Por diferentes razones muchas de ellas fueron siendo dejadas de lado, pero algunas pudieron concretarse, entre ellas dos ciclos de conferencias de divulgación científica en la Sociedad Científica Argentina y una muestra de acuarelas sobre flores y minerales del país, organizada en conjunto con el Museo Minero del SEGEMAR. Los ciclos de conferencias estuvieron referidos a "Mármoles y granitos: de la Historia de la Tierra a la Historia del Arte" y a "Por amor al Arte... y a la Ciencia". Fotografías de las acuarelas y de las muestras de minerales se integraron en un calendario para el año 2013 que fue entregado a todos los participantes en la olimpiada como recuerdo. Para difundir la proximidad del evento e interesar a los medios de comunicación en el 
mismo, un par de semanas antes del comienzo de la Olimpiada se dictó una conferencia sobre "Las relaciones entre la Geología y el Arte" en el salón municipal de la ciudad de Olavarría.

\section{La página web}

Desde principios del año 2012 y en el servidor del Departamento de Ciencias Geológicas (FCEyN-UBA) se instaló la página web del evento, en la que figuraba toda la información necesaria para los potenciales interesados y, finalmente, para los participantes. La misma puede ser aún visitada en la dirección señalada más arriba.

\section{El lema de la $6^{\text {a }}$ IESO}

El lema elegido para esta edición de la Olimpíada fue "Energía, Agua y Minerales para el desarrollo sustentable". La elección estuvo basada en la decisión de hacer visible a la sociedad la importancia del conocimiento geológico, dado el generalizado desconocimiento respecto a muchas características de las fuentes de energía no renovables, las características e importancia del agua subterránea y, también, la existencia de una fuerte posición antiminera en el país. Estas actitudes ultra-ecologistas se sustentan la mayor parte de las veces en un fuerte desconocimiento de la importancia de los minerales en la vida cotidiana y de la necesidad inevitable de su explotación, si bien racional y sustentable, para el desarrollo del país. Con este lema se pensaba, además, que sería más fácil contar con el apoyo de las empresas vinculadas a la minería, cosa que finalmente no ocurrió en la medida esperada.

\section{El logo de la $6^{\mathrm{a}}$ IESO}

Como se ha señalado anteriormente, cada evento define su lema y crea, además su propio logo, en el caso de Argentina se trató de incorporar al mismo no sólo la mirada "científica" académica hacia la Tierra, sino también su significado para las culturas nativas. Es así que, con la colaboración del diseñador $\mathrm{Mg}$. Fabio Massolo se eligió el ícono representado en la Figura 2. El centro del diseño está ocupado por la chakana, adicionándosele los semicírculos concéntricos que representan las distintas esferas que son objeto de estudio (litosfera, hidrósfera, atmósfera y biósfera). La chakana es un símbolo cósmico de origen americano, asociado a la cultura inca pero presente también en otras culturas originarias desde el Ecuador al sur de la Argentina y Chile, y representa las cuatro direcciones del universo, y los distintos planos del mundo tal cual lo entendían los antiguos habitantes de América.

\section{Los premios de la $6^{\text {a }}$ IESO}

En general, cada Olimpíada diseña sus propias medallas y trofeos, así como los diplomas que se entregarán, los cuales, a pesar de tener todos más o menos la misma redacción y ostentar los logos de las instituciones organizadoras, difieren en su composición y, obvia-

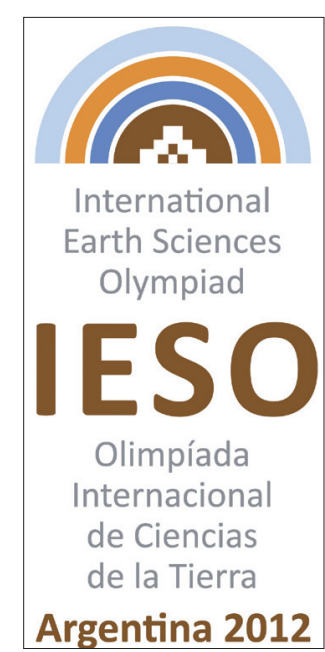

Figura 2. El logo de la $6^{\text {a Olimpíada }}$ Internacional de Ciencias de la Tierra en Argentina mente, en el logo del evento en particular. Para ser entregadas a los ganadores se diseñaron, por lo tanto, las tres medallas tradicionales y un trofeo particular.

\section{Medallas de oro, plata y... cobre}

Las medallas de las IESO no son lo que parecen, pero esto no constituye un engaño, se sabe que es así y que los términos oro, plata y bronce refieren simbólicamente a sus homólogas de competencias más sólidamente financiadas. En el caso de Italia, por ejemplo, el material utilizado fue un compuesto de reciclado de vidrio y se proporcionó a los premiados una explicación acerca del porqué de la elección del material. En el caso de la $6^{a}$ IESO se diseñaron medallas que recordaran los sellos antiguos estampados sobre lacre, utilizándose resina para su confección y pintándoselas con los colores tradicionales, es decir oro, plata y ... bien, resultó que, en el momento de confeccionarse las medallas, no había en el mercado disponibilidad de pintura color bronce, por lo que debió utilizarse un equivalente en color cobre. Durante la presentación se salió del paso expresando, con humor, que se había intentado mantener la pureza de los materiales, ya que el cobre es un metal nativo, pero el cobre no lo es, ya que se trata de una aleación. Todos tomaron a bien la ocurrencia, pero luego se encargaron de solicitar claramente que los diplomas debían decir "Bronze medal" y no "Copper medal"... por las dudas. En la Figura 3 se reproducen las 


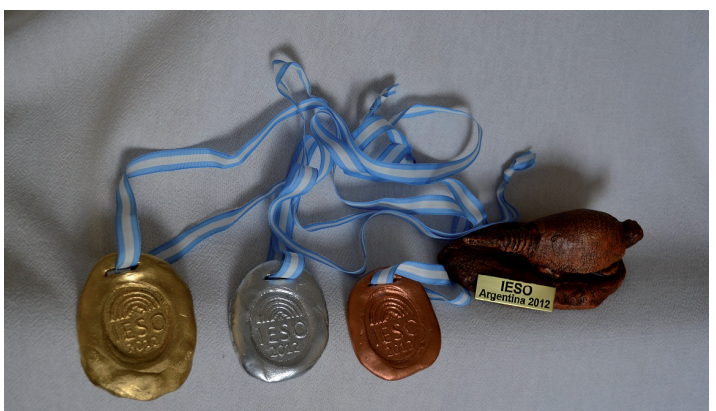

Figura 3. Medallas de oro, plata y cobre/bronce entregadas en la $6^{\mathrm{a}}$ IESO

tres medallas.

\section{Un trofeo que sea a la vez recuerdo y enseñanza}

Con la ayuda del Lic. José Luis Gómez, experto en la reproducción de restos fósiles para docencia y exhibición en museos y muestras (www.galeon. com/paleorama), se diseñó el trofeo que sería entregado a quienes recibieran premios diferentes de las medallas de oro, plata y bronce (también confeccionadas por él). Para ello se eligió una reproducción a escala, realizada en resina, de Panochtus tuberculatus, un fósil sumamente representativo del Cuaternario de las pampas de la Provincia de Buenos Aires, el que se reproduce en el extremo derecho de la Figura 3. Su nombre y características se explicaban en una ficha que se entregó a todos los ganadores del trofeo. La información consignada era la siguiente: "El modelo en escala reproduce uno de los gliptodóntidos más grandes conocidos, ya que llegó a medir $4 \mathrm{~m}$ de largo. Comparte con los otros gliptodontes la característica notable de poseer un voluminoso caparazón rígido del cual asoman la cabeza, los miembros y la cola, que es también acorazada y está protegida en su parte anterior por siete anillos caudales y luego por un tubo caudal rígido. La cabeza presenta un escudete cefálico ("boina”) con una figura central rodeada por varios tubérculos. Fue un animal lento, herbívoro y probablemente de hábitos nocturnos o limitados a algunas horas determinadas del día para evitar el 'recalentamiento' de su armadura. Habitó Sudamérica durante el Pleistoceono medio y superior. Fue descripto por primera vez por German Burmeister en 1866."

\section{Actividades de integración y de formación programadas para los estudiantes participantes}

- Muestra de mapas geológicos: Con unos seis meses de anterioridad a la realización del evento se solicitó a los equipos participantes que diseñaran un poster con un mapa geológico simplificado de su país de origen.

- Muestra de carteles sobre un rasgo geológico destacado de cada uno de los países participantes: Asociado al anterior debía prepararse otro poster en el cual se tomara un rasgo geológico característico o importante del país y se explicaran las razones de su interés.

- Estos pósteres, junto con un tercero en el que se presentaban los integrantes de los equipos fueron expuestos, con gran éxito y entusiasmo de los estudiantes, en el lobby del hotel en el que se hospedaban.

- Visita a Jardín Zoológico y a Museo de Ciencias: La visita al Zoológico fue propuesta en cuanto el mismo, si bien de reducido tamaño, se encuentra especializado en especies autóctonas de la Argentina, lo que resulta no solo un atractivo especial para los extranjeros sino también de alto valor educativo. El Museo de Ciencias si bien modesto, ya que se encuentra en sus primeras etapas, cuenta con una colección de fósiles locales de interés para la temática de la Olimpiada.

- Visita a los estromatolitos y a una cantera: Esta visita se consideró de importancia en cuanto estos afloramientos de estromatolitos, de unos 900 millones de años de antigüedad, son las evidencias de vida más antiguas encontradas hasta ahora en la Argentina. Los mismos se encuentran asociados a una cantera de roca dolomítica, que también fue visitada.

- Visita a una fábrica de ladrillos y tejas: La presencia de calizas y arcillas ha hecho de la zona de Olavarría un centro industrial de producción de cales y cementos, pero también de fabricación de ladrillos y tejas. Se tuvo la oportunidad de visitar la fábrica Cerro Negro, líder en su ramo.

- Visita a la localidad de Sierra Chica. El granito de Sierra Chica es una de las más tradicionales rocas ornamentales del mercado argentino y los participantes fueron recibidos en esa localidad, muy cercana a Olavarría, por los estudiantes de la escuela secundaria local, quienes les mostraron una cantera actualmente abandonada y les ofrecieron una merienda típica.

\section{Crónica del evento}

La llegada de los equipos se produjo entre los días 5 y 7 de Octubre, permaneciendo en la Ciudad 
de Buenos Aires, por sus propios medios, aquéllos que llegaron con anticipación. La organización se ocupó de contratar autobuses que recogieron a los participantes en las cercanías de sus hoteles y en el Aeropuerto Internacional de Ezeiza para que todos pudieran dormir en Olavarría (Ciudad en la que se realizaría el evento) el día domingo. Dos estudiantes de la carrera de Geología de la FCEyN de la UBA viajaron en cada uno de los cuatro autobuses para acompañar y apoyar a aquellos grupos que desconocían el castellano. Estos estudiantes se alojaban también en el hotel de los estudiantes y acompañaron a los grupos durante toda la Olimpíada, ya que los competidores no pueden compartir hotel, ni departir con sus tutores por razones reglamentarias, siendo los guías sus intermediarios.

\section{El primer día}

El día 8 en horas de la mañana se realizó la ceremonia de apertura, en el Teatro Municipal de Olavarría, gentilmente cedido por las autoridades. Como es costumbre en las Olimpíadas, el inicio de la ceremonia estuvo marcado por la entrada de las banderas de los países participantes, a cargo de un miembro de cada delegación y luego se cantó el himno nacional del país organizador. Asistieron a la ceremonia autoridades políticas y administrativas locales, representantes de la IGEO, la IESO y los estudiantes de un colegio local (Nuestra Señora del Rosario) que actuarían como apoyo a los guías. La nota de color la brindaron los representantes de algunas colectividades locales que concurrieron con sus trajes típicos. Para formalizar la apertura de la Olimpíada se dirigió a los concurrentes el Dr. Moon Young Song, representante de la IESO, quién dio la bienvenida a los presentes. A continuación el Coordinador del Comité Organizador Local dio también su bienvenida y agradeció la colaboración de las instituciones y personas que hicieron posible la concreción del evento. El Intendente de Olavarría, Sr. José Eseverri se dirigió a la audiencia para dar también su bienvenida y expresar la importancia que el evento tenía para la ciudad, deseando una buena estadía en la ciudad y mucha suerte a los participantes. La Dra. Sylke Hlwatsh, habló en representación de la IGEO, entidad que preside. Dio a todos la bienvenida y deseo éxito a los participantes. Como cierre del acto los grupos de danzas de la ciudad de Olavarría interpretaron un espectáculo de tango y folklore y al finalizar el mismo con gran éxito entre el público asistente, las autoridades locales ofrecieron un refrigerio con empanadas típicas que fueron celebradas por los miembros de las delegaciones. El evento fue cubierto por los medios locales y la actividad se reflejó en los noticiosos radiales y televisivos de la ciudad y en el periódico del día siguiente. Ya en horas de la tarde comenzó el trabajo de los adultos. Los mentores y observadores de cada equipo debieron abocarse a la preparación de la Prueba Escrita, revisando las preguntas y problemas. Los estudiantes y los invitados, mientras tanto, visitaron el Jardín Zoológico y el Museo de Ciencias locales.

\section{El segundo día}

Durante la mañana del día martes 9 los estudiantes, organizados en grupos de 6 o 7 de diferente nacionalidad, fueron conducidos por los guías al lugar en el que tendría lugar la denominada "Investigación de campo en equipos internacionales". El trabajo, igual para todos los grupos con el propósito de facilitar su evaluación y comparación, consistió en la descripción y análisis de los problemas ambientales que se presentan en el tramo del arroyo Tapalqué a su paso por la zona céntrica de la ciudad de Olavarría. Para ello, además de las observaciones que los participantes podían realizar in situ, se les proporcionaron instrucciones escritas sobre qué hacer y cómo hacerlo, mapas y también gráficos representativos de la pluviometría y los niveles del agua en el arroyo durante tres episodios de inundación de la ciudad, con el propósito de que realizaran un análisis crítico de la situación y propusieran soluciones para la misma. Esta actividad fue entusiastamente seguida por los estudiantes y profesores locales. Luego del almuerzo en el hotel, los grupos analizaron la información recogida y suministrada, escribieron sus informes y, apoyándose en la información gráfica facilitada y en sus propias fotos, prepararon las presentaciones Power Point que el día viernes deberían exponer ante el jurado. Los adultos continuaron durante la mañana reunidos en su hotel preparando la Prueba Escrita.

Luego de la cena, en el hotel de los estudiantes, éstos desplegaron los posters que traían ya preparados de acuerdo a las instrucciones enviadas con mucha anticipación. Uno de estos posters estaba referido a un rasgo geológico característico de su país de origen y otro a los integrantes de cada equipo, en el que quedaran claros sus nombres y apodos y sus rostros, con el objeto de facilitar la difusión de sus países de origen, nombres, intere- 
ses personales, etc. Un jurado de adultos recorrió la muestra para evaluar los posters y proponer los mejores entre ellos para el correspondiente premio. Según todos los comentarios, esta actividad arrojó un resultado excelente y facilitó enormemente la "ruptura del hielo" entre los participantes de los diferentes países. Los miembros del jurado señalaron lo acertado de la idea para promover el acercamiento entre los integrantes de los diferentes equipos. En el otro hotel, mientras tanto, los miembros del Jurado Internacional, que habían trabajado toda la tarde para completar la Prueba Escrita, debieron realizar las traducciones a sus lenguas vernáculas durante la primera parte de la noche y un grupo de voluntarios admirables, liderados por el Dr. Kris Liao, de Taiwán, trabajo hasta la madrugada imprimiendo los cuestionarios para que estuvieran listos para ser distribuidos a los competidores en la mañana.

\section{El tercer día}

El día miércoles 10, luego del desayuno, los competidores fueron guiados al Colegio del Rosario, que gentilmente facilitó sus aulas y la cooperación de sus alumnos y docentes para tomar la Prueba Escrita, el componente más importante de la competencia. Esta actividad finalizó a las 13:00, hora en que los estudiantes se retiraron del lugar para almorzar. Un grupo de profesores locales se ocupó de corregir las Pruebas en función de la grilla de respuestas correctas suministrada por los organizadores. Mientras los competidores se abocaban a la Prueba Escrita, los mentores y observadores se ocuparon de seleccionar y preparar las actividades para la Prueba Práctica. Tanto en la discusión de las preguntas y problemas de la Prueba Teórica como en la selección de las actividades para la Prueba Práctica, más allá de las discusiones lógicas que surgen de diferentes puntos de vista y tradiciones educativas, se puso de manifiesto el espíritu de cooperación de los adultos representantes de todas las naciones participantes. Fue sin duda este espíritu que coloca los intereses de las IESO por encima de los intereses de cualquier equipo o las opiniones de cada uno el que permitió que la Prueba Práctica superara algunos inconvenientes surgidos por deficiencias de infraestructura y equipamiento y resultara exitosa. Desafortunadamente las inclemencias del tiempo impidieron que pudiera llevarse a cabo esa noche la visita al zoo y la observación de las estrellas, en la que colaborarían los astrónomos aficionados locales. Es importante destacar que esta era una inmejorable oportunidad de conocer la Cruz del Sur para los visitantes del Hemisferio Norte. Un grupo de voluntarios parcialmente renovado se ocupó esa noche, nuevamente hasta altas horas de la madrugada, de imprimir las Pruebas Prácticas para ser distribuidas en la mañana del Jueves a los competidores.

\section{El cuarto día}

El día jueves, luego del desayuno y nuevamente en el Colegio del Sagrado Corazón, los estudiantes debieron realizar las mediciones, observaciones y experiencias de la Prueba Práctica. Algunos de los adultos se unieron a los profesores locales para coordinar y supervisar el trabajo de los competidores. El resto de los adultos participaba entre tanto en reuniones vinculadas a temas organizativos y administrativos de la IESO y de la IGEO. El Prof. Chun Yen Chang, de Taiwán presentó una propuesta de modificación de los estatutos de la IESO, pero las conclusiones al respecto fueron postergadas hasta una reunión futura. Luego del almuerzo un grupo representativo de las autoridades de la IESO y países participantes se ocupó de revisar las correcciones de las Pruebas, otorgar puntajes y establecer el orden de méritos para la asignación de medallas y premios. Los jóvenes participantes pudieron distenderse durante la tarde visitando la localidad de Sierra Chica, famosa por la calidad de su granito, de extendido uso como piedra ornamental. Allí fueron calurosamente recibidos por la comunidad escolar que los obsequió con una merienda típica con mate, biscochos caseros y chocolates.

\section{El quinto día}

Finalmente llegó el viernes, último y muy esperado día de la Olimpíada. Por la mañana los grupos internacionales que habían realizado la Investigación de Campo el día martes presentaron los resultados obtenidos frente a los miembros del Jurado y a sus compañeros. Este evento tuvo lugar en el Salón Rivadavia, de la Municipalidad local. La cantidad de público frente a los expositores fue masiva ya que, además de los miembros de los grupos y del jurado, estaban presentes -entre otros- los estudiantes y docentes del Colegio del Rosario que pudieron asistir. Mientras esto ocurría se preparaban los cer- 
tificados, medallas y premios para ser entregados en la ceremonia que tuvo lugar a media tarde en el mismo Salón Rivadavia., con la presencia de las máximas autoridades locales, encabezadas por el Intendente, el Secretario de Cultura y la Secretaria de Turismo; esta última entusiasta e incansable colaboradora en la organización del evento.

El acto de premiación se inició con las alocuciones del Sr. Intendente y la Sra. Secretaria de Turismo quienes destacaron el agradecimiento al Comité Organizador Local por haber elegido la ciudad de Olavarría como sede del evento y el interés que para la ciudad había revestido el mismo. El Dr. Moon Young Song y el Dr. José Sellés-Martínez, coordinador del evento, realizaron los agradecimientos de rigor y a continuación se desarrolló la conferencia de cierre, que estuvo a cargo de la Dra. Andrea Concheiro, geóloga investigadora y docente de la Universidad de Buenos Aires, del Consejo Nacional de Investigaciones Científicas y Técnicas (CONICET) y del Instituto Antártico Argentino (IAA). Justamente a su actividad como geóloga a lo largo de dieciséis campañas, se refirió su presentación, titulada "Aventuras y desventuras de una geóloga en la Antártida”. La presentación fue muy amena y muy bien recibida por la audiencia. Algunos docentes comentaron posteriormente que les había parecido sumamente importante para sus alumnas, que en general desconocían el potencial que una mujer pudiera tener en un ámbito tan hostil como la Antártida.

A continuación comenzó la ceremonia de premiación. La tensión y emoción de los participantes iba en aumento a medida que se otorgaron los diferentes premios y medallas y, lógicamente, alcanzó su máximo al llegarse a las medallas de oro. Pero para ello hubo que ser paciente ya que se comenzó con los premios a los mejores posters, luego a los mejores resultados de la Investigación de Campo y finalmente a las medallas, culminando con el Premio al mejor desempeño. Los premios y certificados fueron entregados por las autoridades y miembros de la IESO y la IGEO presentes. Finalmente para dar fin a la ceremonia el Dr. Moon Young Song y el Dr. José Sellés-Martínez entregaron al Dr. R. Shankar, representante de la India, país en el que se realizará la $7^{\mathrm{a}}$ IESO en 2013, el estandarte de la Olimpíada. Una vez que los aspectos formales fueron completados el clima de la ceremonia cambió radicalmente, ya que toda la ansiedad y la tensión de la semana se transformó en un espectáculo preparado por, y destinado a los estudiantes.
Las presentaciones musicales y los juegos de las delegaciones de Japón (que mostró una serie de juegos tradicionales e invitó a todos a mostrar sus habilidades con ellos), Corea (que sorprendió con un show de luces negras y colores fosforescentes), Indonesia (que deleitó con sus danzas tradicionales) y Taiwán (que fue ovacionado al interpretar el hit del momento "Gangnam Style"). Estas presentaciones sólo fueron interrumpidas cuando las pizzas provistas por la generosidad del Municipio local, atrajeron a todos los participantes. Con mucha pena, llegadas las 22:30 la fiesta debió interrumpirse para que todos volvieran a sus hoteles ya que, entre la medianoche y la madrugada, todos los grupos serían recogidos por los autobuses y conducidos a Ezeiza. Con algo de retraso, debido a la congestión de la ruta, llegaron finalmente los autobuses y cargaron a los estudiantes y los adultos, cansados somnolientos, con enormes cantidades de equipaje... pero felices por las inolvidables experiencias vividas y las medallas conquistadas. Los mentores y observadores con la ilusión, también, de volver a encontrarse el año siguiente en Mysore (India) bajo la generosa hospitalidad del Dr. Shankar y sus colaboradores.

\section{Los premios: los ganadores de esta edición.}

Estando la organización local imbuida de la idea de que la Olimpíada no sólo debe determinar quiénes son los mejores estudiantes entre los participantes, y otorgar a los mismos las respectivas medallas, sino que también debe estimular otros valores más allá de la competición y con la intención de que el mayor número posible de participantes pudiera regresar a su país orgulloso de haberse destacado en alguna actividad en el marco de la Olimpiada se entregaron no sólo las medallas y diplomas oficiales a los ganadores individuales y los trofeos y diplomas oficiales a los ganadores del certamen de Trabajos de Campo, sino también una serie de premios vinculados a las actividades de integración programadas. La lista de premios y premiados es la siguiente:

1. Certamen de pósteres sobre sitios geológicos y la geología de los países participantes: El primer premio correspondió al equipo de Indonesia, integrado por Fenin Rega Randitama, Dovian Emely Suteja, Ratika Benita Nareswari y Thomas Teguh Wijaya; el segundo al equipo de Italia, conformado por MatteoVajente, Lorenzo Terenzi, Andrea Ferrando y Antonio 
Cusano; mientras que el equipo de Australia, compuesto por Clara Tate, Darcy Cathro, Maddi Mellow y Luke Bartlett, recibió el tercer premio.

2. En el marco del trabajo de campo en equipos internacionales, el premio a la mejor presentación correspondió al equipo integrado por Mayara Cardoso Oliveira (Brasil), Nadine Kreutzfeldt (Alemania), Norihiko Nakazato (Japon), Andrei Moldovanu (Rumania), Y1-Shiou Duh (Taiwán), Giulio Salerno (USA) y Varun Ajit Kelkar (India). El equipo acreedor al premio a la mejor cooperación o integración entre ellos fue el de Luke Bartlett (Australia), Hannes Kruse (Alemania), Kento Shimamoto (Japon), Carmen Florea (Rumania), Yi-Ang Peng (Taiwán) y Lyubomyr Romaniv (Ucrania). Los miembros del jurado decidieron otorgar Menciones especiales, una por el modo en que había sido presentada la información, que recayó en Guillaume Prevot (Francia), Ratika Benita Nareswari (Indonesia), Sedong Moon (Corea), Evgenia Tikhova (Rusia), Nuthanun Thanomvajamun (Tailandia) yLindsay Lavee (USA) y la otra por haber llegado a las mejores conclusiones que correspondió a Clara Tate (Australia), Antoine Durieux (Francia), Lorenzo Terenzi (Italia), Maryam Abdullah (Kuwait), Virginia Vilar García (España) y Nazany Vroshchak (Ucrania).

3. Dado que los resultados globales de las pruebas en cada disciplina y en las modalidades teórica y práctica a veces enmascaran logros puntuales destacados, fue propuesto por los organizadores y aceptado por los miembros del jurado internacional, entregar premios a desempeños parciales. Así fueron otorgados el Mejor desempeño masculino en Geosfera a Y1-Shiou Duh (Taiwán), el Mejor desempeño femenino en Geosfera a Bokyung Kim (Corea), el Mejor desempeño masculino en Atmosfera e Hidrosfera a Sedong Moon (Corea), el Mejor Desempeño Femenino en Atmosfera e Hidrosfera a Ratika Benita Nareswari (Indonesia), el Mejor Desempeño Masculino en Astronomía a Chien-Ping Chen (Taiwán) el Mejor desempeño femenino en Astronomía a Bokyung Kim (Corea)

4. Las medallas, máximo galardón del evento en su fase más competitiva, fueron asignadas de acuerdo al siguiente detalle:

5. Se entregó finalmente un premio al Gran Maestro Juvenil de la Olimpíada, que corres- pondió a Donghwan Kim (Corea), quién fue el mejor entre los que recibieron medallas de oro, habiendo obtenido la mejor calificación en el conjunto de pruebas del evento.

\section{Reflexiones finales}

Los países que se proponen a si mismos para la organización de una Olimpíada suelen contar, al momento de hacer la presentación con una cierta seguridad de que contarán con los fondos y el apoyo administrativo para llevar a cabo el evento. Frente a un hecho inesperado, como el sismo y tsunami de Japón, que obliga a cambiar la sede de modo intempestivo, no resulta fácil encontrar una alternativa, ya que el resto de los participantes no ha hecho ninguna gestión al respecto ni cuenta con el tiempo mínimo necesario para llevar a cabo los trámites administrativos y los pedidos de fondos a instituciones públicas y privadas y a las empresas que pudieran ser potenciales patrocinadores. Curiosamente, en estas circunstancias, es más probable que el relevo pueda ser tomado por un país en el cual el esfuerzo personal sea más poderoso que el de las instituciones organizadas. Los países sumamente organizados tienen tiempos de gestión que no pueden ser acortados, mientras que, en aquéllos en los cuales es posible improvisar, las posibilidades se hacen mayores. De todos modos hay que tener en cuenta que, esta diferencia en la mecánica de la organización, tiene sin duda consecuencias en la calidad del evento. No tanto en sus aspectos esenciales, es decir la seriedad de la competencia, sino en la calidad del hospedaje y la comida, por ejemplo, o bien en la diversidad de lugares que pueden ser visitados como parte de las actividades culturales asociadas. A modo de ejemplo puede señalarse que, frente a los más o menos 8 a diez días que suelen durar las Olimpíadas, en la Argentina este lapso debió reducirse a sólo 5 por razones financieras. Del mismo modo, al no contarse con fondos para ello, no se puedo invitar a ninguna delegación, lo que, obviamente, reduce el número de países participantes. Sin embargo, y a pesar de todo ello, y aún sabiendo que parte de ello podía ocurrir, se prefirió no interrumpir la serie continua de Olimpíadas iniciada en el 2007. Al momento de redactarse esta contribución (Febrero del 2014), ha vuelto a ocurrir un hecho similar. Los Estados Unidos que habían asumido el compromiso de organizar la $8^{\text {a }}$ Olimpiada han desistido de hacerlo por razones sumamente com- 
prensibles que no corresponde desarrollar aquí. El pedido de ayuda urgente podría ser atendido esta vez por España, país que deberá informar al respecto en el término de pocos días más. Con toda seguridad, el entusiasmo y conocimiento de los organizadores españoles, vinculados a la Asociación Española para la Enseñanza de las Ciencias de la Tierra (AEPECT), y la experiencia adquirida en la organización de las Olimpíadas Nacionales de Ciencias de la Tierra, les permitirá salir airosos del desafío.

\section{Agradecimientos}

La organización de una olimpíada, aún de reducidas dimensiones, como ésta, exige enormes esfuerzos y la colaboración de muchas personas, que generalmente excede la posibilidad de agradecimiento personalizado a todas ellas. Sin embargo no sería justo dejar de mencionar al Dr. Roberto Greco y a la Prof. Graciela Rastellino por su ayuda en la confección de las preguntas de las pruebas teórica y práctica y a la Sra. María Carmen Calautti por su inestimable cooperación en las tareas de organización y secretaría durante la semana que transcurrió en Olavarría.

Por otra parte, el autor desea agradecer a Celso Dal Ré Carneiro y Pedro Wagner Gonçalves, por su convocatoria y apoyo para participar en los actos de celebración, en el campus Unicamp de los 40 años de experiencia en enseñanza de ciencias de la Tierra del grupo de Brasil, y a todos quienes participaron y colaboraron en la organización de dicho evento.

\section{Referencias}

Kim C.J., Kwak Y. 2004. Eds. Seoul Conference for International Earth Science Olympiad (IESO). Conference Proceedings. Seoul National Univ. 158 p. 